\title{
Negative imaginary synthesis via dynamic output feedback and static state feedback: A Riccati approach
}

DOI:

10.1016/j.automatica.2019.02.055

\section{Document Version}

Accepted author manuscript

Link to publication record in Manchester Research Explorer

\section{Citation for published version (APA):}

Salcan Reyes, G., \& Lanzon, A. (2019). Negative imaginary synthesis via dynamic output feedback and static state feedback: A Riccati approach. Automatica, 104, 220-227. https://doi.org/10.1016/j.automatica.2019.02.055

\section{Published in:}

Automatica

\section{Citing this paper}

Please note that where the full-text provided on Manchester Research Explorer is the Author Accepted Manuscript or Proof version this may differ from the final Published version. If citing, it is advised that you check and use the publisher's definitive version.

\section{General rights}

Copyright and moral rights for the publications made accessible in the Research Explorer are retained by the authors and/or other copyright owners and it is a condition of accessing publications that users recognise and abide by the legal requirements associated with these rights.

\section{Takedown policy}

If you believe that this document breaches copyright please refer to the University of Manchester's Takedown Procedures [http://man.ac.uk/04Y6Bo] or contact uml.scholarlycommunications@manchester.ac.uk providing relevant details, so we can investigate your claim.

\section{OPEN ACCESS}




\title{
Negative imaginary synthesis via dynamic output feedback and static state feedback: A Riccati approach *
}

\author{
Gabriela Salcan-Reyes ${ }^{\mathrm{a}, \mathrm{b}}$, Alexander Lanzon ${ }^{\mathrm{a}}$ \\ ${ }^{a}$ School of Electrical and Electronic Engineering, University of Manchester, Sackville Street, Manchester, M139 PL, UK \\ ${ }^{\mathrm{b}}$ Escuela Superior Politécnica del Litoral, ESPOL, FIEC, Campus Gustavo Galindo Km 30.5 Vía Perimetral, P.O. Box \\ 09-01-5863, Guayaquil, Ecuador
}

\begin{abstract}
This paper is concerned with the study of strictly negative imaginary (SNI) systems and the problem of synthesizing a controller rendering a linear fractional system SNI. First, a set of necessary and sufficient conditions are developed for an SNI lemma without imposing minimality assumptions. The proposed lemma supports both the analysis and synthesis of SNI systems. Then, a set of sufficient conditions, based on the solution of algebraic Riccati equations, is provided for the solution of the SNI synthesis problem. Finally, an illustrative example demonstrates the usefulness of the results given herein.
\end{abstract}

Key words: negative imaginary systems, control system synthesis, output feedback, positive real, robust control, algebraic Riccati equations, uncertain dynamic systems.

\section{Introduction}

Over the past decade, negative imaginary (NI) theory has attracted increasing interest first in robust stability analysis $[10,30,21,18,9]$ and then towards control system synthesis [23,26,20,28,14,13]. Analysis results are now also available for discrete-time systems [6,16,3,17]. Motivated by the stability analysis results of NI systems and the wide range of engineering applications for which NI results are suitable (see [22] for examples), the aim when synthesizing a controller for uncertain systems is to achieve a closed-loop transfer function with an NI or a strictly NI (SNI) property when it is known that the uncertainty is SNI or NI, respectively. Such a problem is called the "NI synthesis problem" [28].

\footnotetext{
* This work was supported by the Engineering and Physical Sciences Research Council (EPSRC) [grant number EP/R008876/1] and the Ecuadorian government's Secretaría de Educación Superior, Ciencia, Tecnología e Innovación (Senescyt). All research data supporting this publication are directly available within this publication.

${ }^{\star \star}$ Early preliminary parts of this work were presented in the 16th European Control Conference, June 2018, Limassol, Cyprus [24] but they are significantly extended here.

Email addresses: Gabriela.Salcan@manchester.ac.uk (Gabriela Salcan-Reyes),

Alexander.Lanzon@manchester.ac.uk (Alexander Lanzon).
}

A fundamental limitation of the controller synthesis methods in $[23,26,20,28]$ is that the closed-loop transfer function is forced to be NI which is not necessarily stable as it can include poles on the $j \omega$-axis [18]. Recently, $[13,14]$ proposed a solution for the Positive Real (PR) synthesis problem which includes both robust stability and performance. $[13,14]$ mention that simple loop transformations transform the NI synthesis problem into their PR framework. However, the transformation in [14] yields a non-proper controller (which must be low-pass filtered) and a non-proper nominal NI feedback loop which looses internal stability when the transformation is reversed from the synthesized PR feedback loop. Also, the transformation in [13] does not yield PR uncertainty for all NI uncertainty, meaning the result would only be applicable to a subclass.

Consequently, an aim of the current article is to provide a controller synthesis method to make a linear fractional closed-loop system both internally stable and SNI. Performance is not considered in this paper. There are two principal difficulties in achieving our aim. As pointed out in $[10,26]$, the first challenge is that an SNI system can only be transformed into a non-strict PR system. This has been a long-standing acute problem specific to NI systems and makes any method for strictly PR synthesis not directly applicable. The second challenge is that there are no (necessary and) sufficient conditions 
in the literature based on non-necessarily minimal statespace realizations to check whether a system is SNI or not. Such a result, referred herein as an SNI lemma, is essential for controller synthesis since minimality of the closed-loop cannot be guaranteed 'a priori'. Therefore, we start by developing an SNI lemma which only requires the assumption of no modes at the origin. This builds on and extends previous results $[30,11,20,29]$. Moreover, the SNI lemma proposed here is based on an algebraic Riccati equation (ARE) with a unique positive semi-definite solution, even though the associated Hamiltonian is singular. The controller synthesis approach presented in this article then requires solution of two AREs. Finally, an illustrative example is given to show the usefulness of the proposed results.

In summary, the main contributions of this paper are as follows: i) fundamental connections are derived between NI systems, spectral factorization, Hamiltonian matrices and AREs; ii) necessary and sufficient conditions are derived for the state-space characterization of SNI systems with non-minimal state-space realizations; iii) sufficient conditions are derived for the solution of the SNI synthesis problem via dynamic output feedback. Along the way, we also capture NI synthesis and static state feedback which have been studied in the literature.

Notation: Let $\mathbb{R}$ and $\mathbb{C}$ denote fields of real and complex numbers, respectively; while $(\cdot)^{m \times n}$ denotes a matrix of dimension $m \times n . \Re(s)$ stands for the real part of $s \in \mathbb{C}$. Let $\mathbb{C}_{<0}=\{s \in \mathbb{C}: \Re(s)<0\}, \mathbb{C}_{\leq 0}=\{s \in \mathbb{C}: \Re(s) \leq$ $0\}, \mathbb{R}_{>0}=\{a \in \mathbb{R}: a>0\}$ and $\mathbb{R}_{\geq 0}=\{a \in \mathbb{R}: a \geq 0\}$. Given $A \in \mathbb{C}^{m \times n}, A^{T}, A^{*}$ and $A^{\dagger}$ denote its transpose, complex conjugate transpose and pseudo-inverse, respectively. Also, $\operatorname{rank}(A)$ denotes the $\operatorname{rank}$ of $A$, while $\operatorname{dim}(\operatorname{ker} A)$ denotes the dimension of the null space of $A$. If $A$ is square, the spectrum of $A$ is denoted by $\sigma(A)$, while $\rho(A)$ denotes the spectral radius of $A$ and $A^{-T}$ is a shorthand notation for $\left(A^{-1}\right)^{T}$. Moreover, $A$ is said to be Hurwitz if $\sigma(A) \in \mathbb{C}_{<0} . I_{n}$ and $0_{n}$ denote the $n \times n$ identity matrix and the $n \times n$ zero matrix, respectively. We will simply use $I$ and 0 when no confusion about the dimensions can arise. $\mathcal{F}_{l}(G, K)$ stands for lower linear fractional transformation of transfer function matrices $G$ and $K . \mathcal{R}$ denotes the set of all real, rational, proper transfer function matrices and $\mathcal{R} \mathcal{H}_{\infty}$ denotes a subset of $\mathcal{R}$ containing all asymptotically stable transfer function matrices. Given $G \in \mathcal{R}, G=\left[\begin{array}{l|l}A & B \\ \hline C & D\end{array}\right]$ is a shorthand for state-space realization $G(s)=C(s I-A)^{-1} B+D$. Moreover, $G^{\sim}(s)=G(-s)^{T}$.

\section{Preliminaries}

NI systems have been defined to include non-rational and non-proper systems $[5,15]$, but we hereinafter restrict attention to NI transfer functions that are real, rational and proper.

Definition 2.1. [18] A real, rational, proper transfer function matrix $G: \mathbb{C} \rightarrow \mathbb{C}^{m \times m}$ is negative imaginary (NI) if the following conditions are satisfied:

(i) $G(s)$ has no poles in $\{s \in \mathbb{C}: \Re(s)>0\}$;

(ii) $j\left[G(j \omega)-G(j \omega)^{*}\right] \geq 0$ for all $\omega \in(0, \infty)$ except for the values of $\omega$ where $j \omega$ is a pole of $G(s)$;

(iii) if $s=j \omega_{0}$ with $\omega_{0} \in(0, \infty)$ is a pole of $G(s)$, then it is a simple pole and $\lim _{s \rightarrow j \omega_{0}}\left(s-j \omega_{0}\right) j G(s)$ is Hermitian and positive semidefinite;

(iv) if $s=0$ is a pole of $G(s)$, then it is at most a double pole and $\lim _{s \rightarrow 0} s^{2} G(s)$ is Hermitian and positive semidefinite.

When the inequality in (ii) is strict and the system is asymptotically stable, we refer to such systems as SNI.

Definition 2.2. [10] A real, rational and proper transfer function $G: \mathbb{C} \rightarrow \mathbb{C}^{m \times m}$ is strictly negative imaginary (SNI) if

(i) $G(s)$ has no poles in $\{s \in \mathbb{C}: \Re(s) \geq 0\}$;

(ii) $j\left[G(j \omega)-G(j \omega)^{*}\right]>0$ for all $\omega>0$.

A subclass of SNI systems is defined below. This subclass is the same as that considered in [11, Theorem 3.3, Theorem 3.4].

Definition 2.3. A real, rational and proper transfer function $G: \mathbb{C} \rightarrow \mathbb{C}^{m \times m}$ is strongly strict negative imaginary (SSNI) if

(i) $G(s)$ is SNI;

(ii) $\lim _{\omega \rightarrow \infty} j \omega\left[G(j \omega)-G(j \omega)^{*}\right]>0$;

(iii) $\lim _{\omega \rightarrow 0^{+}} j \omega^{-1}\left[G(j \omega)-G(j \omega)^{*}\right]>0$.

This definition differs from [5, Theorem 3] since $G$ is not restricted to be symmetric, and the limit in condition (ii) is different from condition (iii) in [5, Theorem 3].

The following technical lemma characterizes the relations among a class of parahermitian rational matrices, AREs and Hamiltonian matrices. This lemma hence allows us to connect SNI systems with important control theory tools developed in the past several decades $[1,7,8]$. The parahermitian matrix considered here is allowed to be singular only at zero frequency to capture essential and peculiar requirements of SNI systems. Note that [31, Theorem 13.19] is not applicable here as the parahermitian is either strictly positive definite at all frequencies or positive semidefinite at all frequencies. That is, it does not allow strict sign for all $\omega>0$ and singular at $\omega=0$.

Lemma 2.4. Let $A, B, P, S, R$ be matrices of compatible dimensions such that $P=P^{T}, R=R^{T}>0$ with $(A, B)$ 
stabilizable. Suppose A has no eigenvalues on the jw-axis. Then, the following statements are equivalent:

(i) The parahermitian rational matrix

$$
\Phi(s)=\left[\begin{array}{c}
(-s I-A)^{-1} B \\
I
\end{array}\right]^{T}\left[\begin{array}{cc}
P & S \\
S^{T} & R
\end{array}\right]\left[\begin{array}{c}
(s I-A)^{-1} B \\
I
\end{array}\right]
$$

satisfies $\Phi(j \omega)>0$ for all $\omega \in \mathbb{R}_{>0} \cup\{\infty\}$ and $\operatorname{det}(\Phi(0))=0$.

(ii) There exists a unique real $X=X^{T}$ such that

$$
A^{T} X+X A-(S+X B) R^{-1}(S+X B)^{T}+P=0
$$

and $\sigma\left(A-B R^{-1}\left(S^{T}+B^{T} X\right)\right) \subset \mathbb{C}_{<0} \cup\{0\}$.

(iii) The Hamiltonian matrix

$$
H=\left[\begin{array}{cc}
A-B R^{-1} S^{T} & -B R^{-1} B^{T} \\
-\left(P-S R^{-1} S^{T}\right) & -\left(A-B R^{-1} S^{T}\right)^{T}
\end{array}\right]
$$

has no jw-axis eigenvalues except at the origin.

PROOF. (i) $\Rightarrow$ (iii) Since $\Phi(j \omega)$ is singular at $\omega=0$ but nonsingular for all $\omega>0$, it follows via [31, Lemma 13.15] that $H$ is singular and has no eigenvalues at $j \omega$ for $\omega>0$.

(iii) $\Rightarrow$ (i) $\Phi(j \omega)$ is nonsingular for all $\omega>0$ and singular at $\omega=0$ by [31, Lemma 13.15]. Now, since $\Phi(j \infty)=$ $R>0$ and $\Phi(j \omega)$ is a continuous function of $\omega$, it follows that $\Phi(j \omega)>0$ for all $\omega>0$.

(i) $\Leftrightarrow$ (ii) Let $F=-R^{-1}\left(S^{T}+B^{T} X\right)$. Condition (i) implies that there exists a unique real $X=X^{T}$ satisfying the ARE in (ii) and $\sigma(A+B F) \subset \mathbb{C}_{\leq 0}$ (via part II of [31, Theorem 13.19]). On the other hand, condition (ii) implies that $\Phi(j \omega) \geq 0$ for all $\omega \in \mathbb{R}_{\geq 0} \cup\{\infty\}$ (via part II of [31, Theorem 13.19]). Then, it only remains to prove that " $\Phi(j \omega)$ is nonsingular on a punctured imaginary axis excluding the origin and $\operatorname{det}(\Phi(0))=0$ if and only if $A+B F$ has no eigenvalues on the $j \omega$-axis except at the origin." Towards this end, observe that there exists a transfer function $M(s)$ such that $\Phi=M^{\sim} R M$, where $M(s)=I-F(s I-A)^{-1} B[31$, Corollary 13.20]. Let $\omega_{0} \in \mathbb{R}$. Because $\Phi(s)$ has full normal rank and $A$ has no eigenvalues on the $j \omega$-axis by assumption, the following statements are equivalent:

- $\operatorname{det}\left(\Phi\left(j \omega_{0}\right)\right)=0$.

- $j \omega_{0}$ is a transmission zero of $M(s)$.

- $M^{-1}(s)=\left[\begin{array}{c|c}A+B F & B \\ \hline F & I\end{array}\right]$ has an observable mode $j \omega_{0}$. [The equivalence follows by [31, Lemma 3.38]]
- $j \omega_{0}$ is an eigenvalue of $A+B F$. [Necessity is obvious. For sufficiency, suppose $j \omega_{0}$ is an eigenvalue of $A+B F$ but is a non-observable mode of $M^{-1}(s)$. Then, there exists an $0 \neq x \in \mathbb{C}^{n}$ such that $(A+$ $B F) x=j \omega_{0} x$ and $F x=0$. This implies that $A$ has eigenvalues on the $j \omega$ axis, which is a contradiction.]

This concludes the proof.

\section{SNI lemma with non-minimal state-space re- alizations}

A set of conditions used to check if a system is SNI or not is known as the SNI lemma. The earliest versions of the SNI lemma considered minimal state-space realizations based on linear matrix inequalities (LMIs) [30] or an ARE [20]. Yet, an SNI lemma without the assumption of minimal state-space realization is essential for controller synthesis. While $[11,29]$ have relaxed the minimality assumption, [11] imposes conditions at infinity and at zero frequency which restrict the SNI class to the subclass known as SSNI. Since we are interested in providing a solution to the complete SNI synthesis problem, the SSNI Lemma [11] cannot be used here. On the other hand, in [29, Corollary 4] the system matrix is required to be Hurwitz as an assumption instead of being implied by the conditions, therefore it is not useful, on its own, to guarantee the internal stability of the resulting closedloop. Also, [26] provides an NI Lemma without minimal assumptions based on LMIs, but the system is allowed to be marginally stable which is not desirable. Here, we provide an SNI Lemma which guarantees not only the SNI property but also the stability of the system under the mild assumption of $A$ being nonsingular. Moreover, the proposed SNI lemma generalizes some of the results in $[20,19]$ since minimal state-space realization is not assumed here.

Theorem 3.1 (SNI Lemma). Let $\left[\begin{array}{l|l}A & B \\ \hline C & D\end{array}\right]$ be a statespace realization of the real, rational, proper transfer function $G: \mathbb{C} \rightarrow \mathbb{C}^{m \times m}$ with $R=C B+(C B)^{T}>0$. Then, the following statements are equivalent:

(i) $G(s)$ is SNI and $A$ is Hurwitz;

(ii) $D=D^{T}$, A is Hurwitz and the Hamiltonian matrix

$$
H=\left[\begin{array}{cc}
A-B R^{-1} C A & B R^{-1} B^{T} \\
-A^{T} C^{T} R^{-1} C A-\left(A-B R^{-1} C A\right)^{T}
\end{array}\right](1)
$$

has no $j \omega$-axis eigenvalues except at the origin; (iii) $D=D^{T}$, $\operatorname{det}(A) \neq 0$ and there exists a real $X=$ 
$X^{T} \geq 0$ such that

$$
\begin{aligned}
& A^{T} X+X A \\
& \quad+\left(C A-B^{T} X\right)^{T} R^{-1}\left(C A-B^{T} X\right)=0
\end{aligned}
$$

and $\sigma\left(A-B R^{-1}\left(C A-B^{T} X\right)\right) \subset \mathbb{C}_{<0} \cup\{0\}$;

(iv) $D=D^{T}, A$ is Hurwitz and there exist a real $X=$ $X^{T}$ and a real $L$ such that

$$
\begin{aligned}
A^{T} X+X A & =-L^{T} L, \\
C & =-B^{T} A^{-T} X
\end{aligned}
$$

and $\operatorname{rank}(M(j \omega))=m$ for all $\omega \in \mathbb{R}_{>0}$, where $M(s)=L A^{-1}(s I-A)^{-1} B$.

PROOF. Let $F(s)=s(G(s)-D)$ and

$$
\begin{aligned}
\Phi(s) & =F(s)+F^{\sim}(s) \\
& =\left[\begin{array}{c}
-(s I+A)^{-1} B \\
I
\end{array}\right]^{T}\left[\begin{array}{cc}
0 & (C A)^{T} \\
C A & R
\end{array}\right]\left[\begin{array}{c}
(s I-A)^{-1} B \\
I
\end{array}\right] .
\end{aligned}
$$

Note that $G(s)$ is SNI if and only if $D=D^{T}, F(s) \in$ $\mathcal{R H}_{\infty}, F(0)=0$ and $\Phi(j \omega)>0$ for all $\omega$ in $\mathbb{R}_{>0}$ by $[11$, Lemma 3.1]. Then, condition (i) is equivalent to

(i') $D=D^{T}, A$ is Hurwitz and $\Phi(j \omega)>0$ for all $\omega \in$ $\mathbb{R}_{>0}$.

(i') $\Leftrightarrow$ (ii) This follows by Lemma 2.4 on noting that assumptions of Lemma 2.4 are satisfied, $\operatorname{det}(\Phi(0))=0$, $\Phi(\infty)=R>0$ and

$$
\sigma(H)=\sigma\left(\left[\begin{array}{cc}
A-B R^{-1} C A & -B R^{-1} B^{T} \\
A^{T} C^{T} R^{-1} C A & -\left(A-B R^{-1} C A\right)^{T}
\end{array}\right]\right) .
$$

(ii) $\Rightarrow$ (iii) This follows from Lemma 2.4 on noting that $X \geq 0$ since $A$ is Hurwitz and $A^{T} X+X A \leq 0$ (from $(2))$.

(iii) $\Rightarrow$ (ii) We will show that $X$ is unique and $A$ is Hurwitz, so the implication follows by Lemma 2.4. To achieve this, let us first assume that $A$ is Hurwitz. Then, a simple Popov-Belevitch-Hautus test [31] shows that the pair $\left(A-B R^{-1} C A, B R^{-1} B^{T}\right)$ is stabilizable. This guarantees the uniqueness of $X[31,2,8]$. The proof is completed by showing that $\left(C A-B^{T} X, A\right)$ is detectable, because it implies $A$ is Hurwitz (by noting that $X \geq 0$ and $A^{T} X+X A \leq 0$ are satisfied by assumption). So, let us assume, to the contrary, that the pair $\left(C A-B^{T} X, A\right)$ is not detectable. Then, there exist $0 \neq x \in \mathbb{C}^{n}$ and $\Re(\lambda) \geq 0$ such that $A x=\lambda x,\left(C A-B^{T} X\right) x=0$ and $\left(\bar{A}-B R^{-1}\left(C A-B^{T} X\right)\right) x=\lambda x$. Therefore, $\lambda$ is also an eigenvalue of $A-B R^{-1}\left(C A-B^{T} X\right)$ which implies that $\lambda=0$. This contradicts $\operatorname{det} A \neq 0$. Therefore, $\left(C A-B^{T} X, A\right)$ is detectable.

(iii) $\Rightarrow$ (iv) $A$ is Hurwitz, as shown above. Also, it follows from [24, Lemma 2] that (3) and (4) hold. Furthermore, $\Phi(s)=-s^{2} M^{\sim}(s) M(s)$ is easily verified by the use of $(3),(4)$ and by routine algebra. Finally, $\operatorname{rank}(M(j \omega))=$ $m$ for all $\omega \in \mathbb{R}_{>0}$ since $\Phi(j \omega)>0$ for all $\omega \in \mathbb{R}_{>0}$ (by the equivalence between (iii) and (i')).

(iv) $\Rightarrow($ i') This was proved in [29, Corollary 4$]$.

Remark 3.2. Note that when any of these conditions hold, the almost stabilizing solution to the ARE (2) is unique, as shown in the proof of necessity (iii) $\Rightarrow$ (ii). Also, the partial multiplicities (i.e. the sizes of the Jordan blocks to which an eigenvalue belongs) of the zero eigenvalues of the Hamiltonian (1) are all even [2,8]. Furthermore, assumption $R>0$ allows us to establish the necessity of (iv), but this assumption can be removed when only sufficiency of (iv) is of interest as shown in $[29]$.

The importance of Theorem 3.1 can be summarized as follows: 1) The SNI property can be easily and directly tested by checking the spectrum of the associated Hamiltonian which depends only on the state-space matrices (non-necessarily minimal). 2) Condition (iii) is useful for synthesis as shown in section 5. 3) Condition (iv) provides a connection with existing literature, however, it is not desired for synthesis because it involves a rank condition that results in a non-convex problem which is hard to solve.

\section{A strongly SNI Lemma with non-minimal state-space realization}

Interestingly, for SSNI systems the spectral properties of the Hamiltonians allow us to transform an ARE with an almost stabilizing solution into an ARE that has the same solution but being stabilizing for this new ARE. Consequently, we provide an SSNI lemma based on Hamiltonians and an ARE with better numerical properties. The ARE changing procedure was motivated by the double-shift technique in [2]. Notice that the SSNI lemma in [11] is based on LMIs which is complementary to the SSNI lemma below. Moreover, contrary to [11] we do not impose any controllability or observability condition on the state-space realization, only the mild assumption of $A$ being Hurwitz.

Lemma 4.1 (SSNI Lemma). Let $\left[\begin{array}{c|c}A & B \\ \hline C & D\end{array}\right]$ be a statespace realization of the real, rational, proper transfer function $G: \mathbb{C} \rightarrow \mathbb{C}^{m \times m}$ with $R=C B+(C B)^{T}>0$. Then, the following statements are equivalent: 
(i) $G(s)$ is SSNI with A Hurwitz;

(ii) $D=D^{T}$, $\operatorname{det}(A) \neq 0$ and there exists a real $X=$ $X^{T} \geq 0$ such that (2) holds and the matrix $A-$ $B R^{-1}\left(C A-B^{T} X\right)$ has all its eigenvalues in $\mathbb{C}_{<0}$ except $m$ eigenvalues at the origin;

(iii) $D=D^{T}$, $\operatorname{det}(A) \neq 0$ and there exists a real $X=$ $X^{T} \geq 0$ such that $C=-B^{T} A^{-T} X$,

$$
\widetilde{A}^{T} X+X \widetilde{A}+X \widetilde{B} X+\widetilde{C}=0,
$$

and $\sigma(\widetilde{A}+\widetilde{B} X) \subset \mathbb{C}_{<0}$, where for any $\eta<0$,

$$
\begin{aligned}
& \widetilde{A}=A-B R^{-1} C A+\eta A^{-1} B\left(B^{\dagger} A-C\right), \\
& \widetilde{B}=B R^{-1} B^{T}-\eta A^{-1} B B^{T} A^{-T}, \\
& \widetilde{C}=A^{T} C^{T} R^{-1} C A-\eta\left[C^{T}\left(C-B^{\dagger} A\right)-\left(B^{\dagger} A\right)^{T} C\right] .
\end{aligned}
$$

Furthermore, the matrix $X$ in conditions (ii) and (iii) is the same.

PROOF. Let $H$ as in $(1), S=\left[\left(A^{-1} B\right)^{T}-C\right]^{T}, W=$ $\left[\left(A^{-2} B\right)^{T} C A^{-1}\right]^{T}, \Lambda=A-B R^{-1}\left(C A-B^{T} X\right)$ and $E=\left[\begin{array}{cc}0 & I \\ -I & 0\end{array}\right]$. Note that the columns of $A^{-1} B, S$ and $W$ are linearly independent because $\operatorname{det}(R) \neq 0$. Moreover, a simple calcultion shows that $H S=0, S^{T} E H=0$.

(i) $\Leftrightarrow$ (ii) Observe that Theorem 3.1 already establishes part of this equivalence. Then, $C=-B^{T} A^{-T} X$ and $K=\lim _{\omega \rightarrow 0^{+}} \omega^{-1} j\left[G(j \omega)-G(j \omega)^{*}\right]=C A^{-2} B+$ $B^{T}\left(A^{-T}\right)^{2} C^{T} \geq 0$. Hence, it remains to prove that $\gamma=$ $\operatorname{dim}(\operatorname{ker} K)=0$ if and only if $H$ has $2 m$ zero eigenvalues (by noting that $\left[\begin{array}{cc}I & 0 \\ -X & I\end{array}\right] H\left[\begin{array}{ll}I & 0 \\ X & I\end{array}\right]=\left[\begin{array}{cc}\Lambda & B R^{-1} B^{T} \\ 0 & -\Lambda^{T}\end{array}\right]$ ). To this end, observe that $K=S^{T} E W$ and there exists a nonsingular $P$ such that $P^{T} K P=\left[\begin{array}{ll}I & 0 \\ 0 & 0_{\gamma}\end{array}\right]$. Thus, $\gamma$ columns of $W P$ are orthogonal to $\gamma$ columns of ESP and clearly, the columns of $W P$ are right generalized eigenvectors of $H$ (i.e. $H W P=S P$ ) while the columns of $E S P$ are left eigenvectors corresponding to zero eigenvalues (i.e. $\left.(E S P)^{T} H=0\right)$. Then, in view of $[2$, Remark A.8], $\gamma$ zero eigenvalues of $H$ have partial multiplicity strictly greater than two. Hence, $\gamma=0$ if and only if $H$ has $2 m$ zero eigenvalues.

(ii) $\Leftrightarrow$ (iii) Let $\widetilde{H}$ be the Hamiltonian associated with the ARE (5). First, note that (ii) implies (4) by Theorem 3.1. Also, notice that $\widetilde{H}=H+\eta S F^{T}+\eta Q S^{T} E$, where $F=\left[\begin{array}{ll}B^{\dagger} A & 0\end{array}\right]^{T}, Q=\left[-\left(A^{-1} B\right)^{T} C-B^{\dagger} A\right]^{T}$.
Then, $S=\left[\begin{array}{ll}I & X\end{array}\right]^{T} A^{-1} B, S^{T} E\left[\begin{array}{ll}I & X\end{array}\right]^{T}=0$ and $S F^{T}\left[\begin{array}{ll}I & X\end{array}\right]^{T}=\left[\begin{array}{ll}I & X\end{array}\right]^{T} A^{-1} B B^{\dagger} A$. Consequently, $\widetilde{H}\left[\begin{array}{ll}I & X\end{array}\right]^{T}=\left[\begin{array}{ll}I & X\end{array}\right]^{T} \widetilde{\Lambda}$ if and only if $H\left[\begin{array}{ll}I & X\end{array}\right]^{T}=$ $\left[\begin{array}{ll}I & X\end{array}\right]^{T} \Lambda$, where $\widetilde{\Lambda}=\Lambda+\eta A^{-1} B B^{\dagger} A$. In other words, $X=X^{T} \geq 0$ is a solution to (2) if and only if $X=X^{T} \geq 0$ is a solution to (5) and (4) holds. Next, it only remains to show that $\widetilde{\Lambda}$ is Hurwitz if and only if $\Lambda$ has all its eigenvalues in $\mathbb{C}_{<0}$ except $m$ eigenvalues at the origin. To this end, note that $\operatorname{rank}(\Lambda)=n-m$ because $\operatorname{rank}(\Lambda)=\operatorname{rank}\left(\Lambda A^{-1}\right)$ and the eigenvalues of $B R^{-1}\left(C-B^{T} X A^{-1}\right)$ are $m$ at unity together with $n-m$ eigenvalues at zero, obtained by observing that the non-zero eigenvalues of $B R^{-1}\left(C-B^{T} X A^{-1}\right)$ are the same as those of $R^{-1}\left(C B-B^{T} X A^{-1} B\right)=I_{m}$. This yields $\operatorname{dim}(\operatorname{ker} \Lambda)=m$ and $\operatorname{ker} \Lambda=\operatorname{Im}\left(V_{1}\right)$, where $V_{1}=A^{-1} B$. If we now assume that $\Lambda$ has $m+k$ eigenvalues at the origin, then there exist matrices $M \in \mathbb{C}^{m \times k}$ and $J \in \mathbb{C}^{n-(m+k) \times n-(m+k)}$ and full column rank matrices $V_{2} \in \mathbb{C}^{n \times k}, V_{3} \in \mathbb{C}^{n \times n-(m+k)}$ such that $\left[\begin{array}{lll}V_{1} & V_{2} & V_{3}\end{array}\right]$ is nonsingular and $\Lambda\left[\begin{array}{lll}V_{1} & V_{2} & V_{3}\end{array}\right]=$ $\left[\begin{array}{lll}V_{1} & V_{2} & V_{3}\end{array}\right]\left[\begin{array}{ccc}0_{m} & M & 0 \\ 0 & 0_{k} & 0 \\ 0 & 0 & J\end{array}\right]$. Consequently, $\widetilde{\Lambda}\left[\begin{array}{lll}V_{1} & V_{2} & V_{3}\end{array}\right]=$ $\left[\begin{array}{lll}V_{1} & V_{2} & V_{3}\end{array}\right]\left[\begin{array}{ccc}\eta I_{m} & M+\eta V_{1}^{\dagger} V_{2} & \eta V_{1}^{\dagger} V_{3} \\ 0 & 0_{k} & 0 \\ 0 & 0 & J\end{array}\right]$. Thus, $\widetilde{\Lambda}$ is Hurwitz if and only if $J$ is Hurwitz and $k=0$.

\section{SNI control problem}

Consider the uncertain linear system depicted in Fig. 1, where the uncertainty $\Delta(s)$ is assumed to be NI and the generalized plant $G(s)$ is described by

$$
\begin{aligned}
& \dot{x}=A x+B_{1} w+B_{2} u, \\
& z=C_{1} x+D_{11} w+D_{12} u, \\
& y=C_{2} x+D_{21} w+D_{22 u},
\end{aligned}
$$

where $x$ is the state of the plant, $w$ is the disturbance acting on the system, $u$ is the control input, $z$ is the controlled output signal, $y$ is the measurement output, $A \in \mathbb{R}^{n \times n}, B_{1} \in \mathbb{R}^{n \times m} B_{2} \in \mathbb{R}^{n \times q}, C_{1} \in \mathbb{R}^{m \times n}, C_{2} \in$ $\mathbb{R}^{p \times n}, D_{11} \in \mathbb{R}^{m \times m}, D_{12} \in \mathbb{R}^{m \times q}, D_{21} \in \mathbb{R}^{p \times m}, D_{22} \in$ $\mathbb{R}^{p \times q}, q \leq m$ and $p \leq m$.

Our aim is to construct a controller $K(s)$ such that the closed-loop system $\mathcal{F}_{l}(G, K)$ is SNI and the associated linear fractional interconnection is internally stable. 


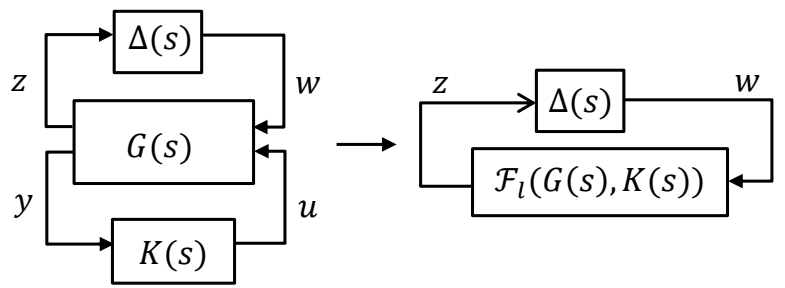

Fig. 1. Feedback control of an uncertain system

\subsection{Static state-feedback}

When all the system states are available, a statefeedback controller can render the closed-loop system SNI, as shown in the following result.

In this case, the generalized plant $G(s)$ is described by

$$
\begin{aligned}
\dot{x} & =A x+B_{1} w+B_{2} u \\
z & =C_{1} x+D_{11} w \\
y & =x
\end{aligned}
$$

and the static state-feedback control law is given by $u=$ $F x$.

Theorem 5.1. Consider the generalized plant $G(s)$ in (7) where $C_{1} B_{2}$ is full column rank, and $\left(A, B_{2}\right)$ is stabilizable. Let $R=C_{1} B_{1}+B_{1}^{T} C_{1}^{T}>0$ and suppose that

(i) $D_{11}=D_{11}^{T}$;

(ii) there exists a real $X \geq 0$ such that

$$
A_{x}^{T} X+X A_{x}+X R_{x} X+Q_{x}=0
$$

where

$$
\begin{aligned}
A_{x}= & \left(I-B_{1} R^{-1} C_{1}\right)\left[I-B_{2}\left(B_{2}^{T} C_{1}^{T} R^{-1} C_{1} B_{2}\right)^{-1}\right. \\
& \left.B_{2}^{T} C_{1}^{T} R^{-1} C_{1}\right] A, \\
R_{x}= & B_{1} R^{-1} B_{1}^{T}-\left(I-B_{1} R^{-1} C_{1}\right) B_{2}\left(B_{2}^{T} C_{1}^{T} R^{-1}\right. \\
& \left.C_{1} B_{2}\right)^{-1} B_{2}^{T}\left(I-B_{1} R^{-1} C_{1}\right)^{T}, \\
Q_{x}= & A^{T} C_{1}^{T} R^{-1}\left[R-C_{1} B_{2}\left(B_{2}^{T} C_{1}^{T} R^{-1} C_{1} B_{2}\right)^{-1} B_{2}^{T}\right. \\
& \left.C_{1}^{T}\right] R^{-1} C_{1} A .
\end{aligned}
$$

Then, the state-feedback controller $u=F x$ generates an NI closed-loop system

$$
\left[\begin{array}{c|c}
A+B_{2} F & B_{1} \\
\hline C_{1} & D_{11}
\end{array}\right],
$$

where

$$
\begin{aligned}
F= & -\left(B_{2}^{T} C_{1}^{T} R^{-1} C_{1} B_{2}\right)^{-1} B_{2}^{T}\left[X+C_{1}^{T} R^{-1}\right. \\
& \left.\left(C_{1} A-B_{1}^{T} X\right)\right] .
\end{aligned}
$$

Moreover, if in addition $\sigma\left(A_{x}+R_{x} X\right) \subset \mathbb{C}_{<0} \cup\{0\}$ and $\operatorname{det}\left(A+B_{2} F\right) \neq 0$, the closed-loop system (12) is $S N I$ with $A+B_{2} F$ Hurwitz.
PROOF. Let $A_{F}=A+B_{2} F$. Then,

$$
\begin{aligned}
0= & A_{x}^{T} X+X A_{x}+X R_{x} X+Q_{x} \\
= & A^{T} X+X A+\left(C_{1} A-B_{1}^{T} X\right)^{T} R^{-1}\left(C_{1} A-B_{1}^{T} X\right) \\
& -\left[X+\left(C_{1} A-B_{1}^{T} X\right)^{T} R^{-1} C_{1}\right] B_{2}\left(B_{2}^{T} C_{1}^{T} R^{-1}\right. \\
& \left.C_{1} B_{2}\right)^{-1} B_{2}^{T}\left[X+\left(C_{1} A-B_{1}^{T} X\right)^{T} R^{-1} C_{1}\right]^{T} \\
= & A_{F}^{T} X+X A_{F}+\left(C_{1} A_{F}-B_{1}^{T} X\right)^{T} R^{-1}\left(C_{1} A_{F}\right. \\
& \left.-B_{1}^{T} X\right) .
\end{aligned}
$$

Hence, (12) is NI by [24, Lemma 2]. Moreover note that,

$$
\begin{aligned}
& A_{x}+R_{x} X \\
&=\left(I-B_{1} R^{-1} C_{1}\right) A+B_{1} R^{-1} B_{1}^{T} X-\left(I-B_{1} R^{-1} C_{1}\right) \\
& B_{2}\left(B_{2}^{T} C_{1}^{T} R^{-1} C_{1} B_{2}\right)^{-1} B_{2}^{T}\left[C_{1}^{T} R^{-1} C_{1} A+\right. \\
&\left.\left(I-B_{1} R^{-1} C_{1}\right)^{T} X\right] \\
&=\left(I-B_{1} R^{-1} C_{1}\right) A+B_{1} R^{-1} B_{1}^{T} X+\left(I-B_{1} R^{-1} C_{1}\right) \\
& B_{2} F \\
&= A_{F}-B_{1} R^{-1}\left(C_{1} A_{F}-B_{1}^{T} X\right) .
\end{aligned}
$$

Thus, on exploiting the fact that $X \geq 0$ satisfies (8) together with $\sigma\left(A_{x}+R_{x} X\right) \subset \mathbb{C}_{<0} \cup\{0\}$ and $\operatorname{det}(A+$ $\left.B_{2} F\right) \neq 0$, the closed-loop (12) is SNI with $A+B_{2} F$ Hurwitz by Theorem 3.1.

Remark 5.2. Note that when $A$ is nonsingular the columns of $A^{-1} B_{2}$ belongs to $\operatorname{ker} A_{x} \cap \operatorname{ker} Q_{x}$. On the other hand, when $A$ is singular there exists $0 \neq v \in \mathbb{R}^{n}$ such that $A v=0$, thus $v \in \operatorname{ker} A_{x} \cap \operatorname{ker} Q_{x}$. Consequently, ker $A_{x} \cap \operatorname{ker} Q_{x}$ is not an empty set. Then, it follows that the ARE (8) has a singular Hamiltonian and its solution may not be unique. However, we can find some of these solutions by following the procedure in [12]. Singular Hamiltonians arise in NI controller synthesis.

Remark 5.3. The assumption that $\left(A, B_{2}\right)$ is stabilizable is not needed to design a feedback gain $K$ such that $\mathcal{F}_{l}(G, K)$ is NI. This assumption is however required for $A+B_{2} F$ to be Hurwitz.

\subsection{Dynamic output feedback}

Consider the generalized plant $G(s)$ in (6). The controller $K: \mathbb{C} \rightarrow \mathbb{C}^{q \times p}$ is described by

$$
\begin{aligned}
\dot{x}_{k} & =A_{k} x_{k}+B_{k} y \\
u & =C_{k} x_{k}+D_{k} y
\end{aligned}
$$

where $x_{k}$ is the state of the controller, $A_{k} \in \mathbb{R}^{k \times k}, B_{k} \in$ $\mathbb{R}^{k \times p}, C_{k} \in \mathbb{R}^{q \times k}$ and $D_{k} \in \mathbb{R}^{q \times p}$.

Suppose the generalized plant state-space matrices in (6) satisfy the following assumptions:

(A1) $\left(A, B_{2}\right)$ is stabilizable and $\left(C_{2}, A\right)$ is detectable; 
(A2) $C_{1} B_{2}$ is full column rank, $D_{21}$ has full row rank;

(A3) $D_{12}=0$ and $D_{22}=0$;

(A4) $C_{1} B_{1}+B_{1}^{T} C_{1}^{T}>0$.

Assumption (A1) is necessary for internal stability. Assumption (A2) is analogous to the corresponding assumption in LQG $/ \mathcal{H}_{\infty} / \mathrm{PR}$ synthesis $[4,27]$ for the system $s\left(G(s)-D_{11}\right)=\left[\begin{array}{c|cc}A & B_{1} & B_{2} \\ \hline C_{1} & C_{1} B_{1} & C_{1} B_{2} \\ C_{2} & D_{21} & D_{22}\end{array}\right]$. The assumption that $C_{1} B_{2}$ is full column rank in (A2) means that the transfer function from $u$ to $z$ has relative degree of one. The assumptions in (A3) can easily be relaxed as shown in [25, Lemma 5]. Assumption (A4) is necessary for the synthesis method presented in the following result.

Theorem 5.4. Consider the generalized plant $G(s)$ in (6) and let the assumptions (A1) to (A4) hold. Let $R=$ $C_{1} B_{1}+B_{1}^{T} C_{1}^{T}$ and suppose that

(i) $D_{11}=D_{11}^{T}$;

(ii) there exists a real $X \geq 0$ such that $A_{x}^{T} X+X A_{x}+$ $X R_{x} X+Q_{x}=0$, where $A_{x}, R_{x}$ and $Q_{x}$ are defined in (9), (10) and (11), respectively;

(iii) there exists a real $Y \geq 0$ such that $A_{y} Y+Y A_{y}^{T}+$ $Y R_{y} Y+Q_{y}=0$ and $\sigma\left(A_{y}+Y R_{y}\right) \subset \mathbb{C}_{<0}$, where

$$
\begin{aligned}
A_{y}= & \left(I-B_{1} R^{-1} C_{1}\right) A-B_{1} R^{-1} D_{21}^{T} \\
& \left(D_{21} R^{-1} D_{21}^{T}\right)^{-1}\left(C_{2}-D_{21} R^{-1} C_{1} A\right), \\
R_{y}= & A^{T} C_{1}^{T} R^{-1} C_{1} A-\left(C_{2}-D_{21} R^{-1} C_{1} A\right)^{T} \\
& \left(D_{21} R^{-1} D_{21}^{T}\right)^{-1}\left(C_{2}-D_{21} R^{-1} C_{1} A\right), \\
Q_{y}= & B_{1} R^{-1}\left[R-D_{21}^{T}\left(D_{21} R^{-1} D_{21}^{T}\right)^{-1} D_{21}\right] R^{-1} B_{1}^{T} ;
\end{aligned}
$$

(iv) $\rho(Y X)<1$.

Then, the closed-loop system $\mathcal{F}_{l}(G, K)$ is NI when $K(s)$ is given by (15) with $A_{k}=A+B_{2} F+(I-$ $Y X)^{-1} L C_{2}+\Psi, B_{k}=-(I-Y X)^{-1} L, C_{k}=F$ and $D_{k}=0$, where $F$ is defined as in (13), $L=$ $-\left[Y C_{2}^{T}+\left(B_{1}^{T}-C_{1} A Y\right)^{T} R^{-1} D_{21}^{T}\right]\left(D_{21} R^{-1} D_{21}^{T}\right)^{-1}$, $\Psi=-\left[B_{1}+(I-Y X)^{-1} L D_{21}\right] R^{-1}\left[C_{1}\left(A+B_{2} F\right)-\right.$ $\left.B_{1}^{T} X\right]$. Moreover, if $\sigma\left(A_{x}+R_{x} X\right) \subset \mathbb{C}_{<0} \cup\{0\}$ and $\operatorname{det}\left(\left[\begin{array}{cc}A+B_{2} F & -B_{2} F \\ -\Psi & A+\Psi-B_{k} C_{2}\end{array}\right]\right) \neq 0$, then $\mathcal{F}_{l}(G, K)$ is SNI and the associated linear fractional interconnection is internally stable.

PROOF. This proof follows the results given for strictly PR (SPR) systems in [27], but important modifications are made to allow the synthesis of SNI systems. Using the specified state-space realizations for $G(s)$ and $K(s)$ in (6) and (15), respectively, the closed-loop system $\mathcal{F}_{l}(G, K)$ has the following state-space realization, with $\left[x^{T} x^{T}-x_{k}^{T}\right]^{T}$ as the state vector

$$
\left[\begin{array}{c|c}
A_{c} & B_{c} \\
\hline C_{c} & D_{c}
\end{array}\right]=\left[\begin{array}{cc|c}
A+B_{2} F & -B_{2} F & B_{1} \\
-\Psi & A+\Psi-B_{k} C_{2} & B_{1}-B_{k} D_{21} \\
\hline C_{1} & 0 & D_{11}
\end{array}\right] .
$$

We will show first that conditions (i) to (iv) imply that there exists a real $\mathcal{X}=\mathcal{X}^{T} \geq 0$ such that

$$
\begin{aligned}
\mathcal{Q}(\mathcal{X})= & A_{c}^{T} \mathcal{X}+\mathcal{X} A_{c} \\
& +\left(C_{c} A_{c}-B_{c}^{T} \mathcal{X}\right)^{T} R^{-1}\left(C_{c} A_{c}-B_{c}^{T} \mathcal{X}\right)=0
\end{aligned}
$$

which, according to [24, Lemma 2], would guarantee that the closed-loop system is NI. Then, we will show that

$$
\sigma\left(A_{c}-B_{c} R^{-1}\left(C_{c} A_{c}-B_{c}^{T} \mathcal{X}\right)\right) \subset \mathbb{C}_{<0} \cup\{0\}
$$

holds when $\sigma\left(A_{x}+R_{x} X\right) \subset \mathbb{C}_{<0} \cup\{0\}$, because (16) and (17) would guarantee, when $\operatorname{det}\left(A_{c}\right) \neq 0$, that the closed-loop system is SNI with $A_{c}$ Hurwitz via Theorem 3.1.

Towards this end, let $A_{F}=A+B_{2} F$. Then, by Theorem 5.1, conditions (i) and (ii) imply that (14) holds. Next, let $A_{z}=A_{y}+Q_{y} X$ and $R_{z}=X A_{z}+A_{z}^{T} X-$ $X Q_{y} X+R_{y}$, so

$$
\begin{aligned}
0= & A_{y} Y+Y A_{y}^{T}+Y R_{y} Y+Q_{y} \\
= & \left(A_{z}-Q_{y} X\right) Y+Y\left(A_{z}-Q_{y} X\right)^{T}+Y\left(X Q_{y} X-\right. \\
& \left.X A_{z}-A_{z}^{T} X+R_{z}\right) Y+Q_{y} \\
= & (I-Y X) A_{z} Y+Y A_{z}^{T}(I-X Y)+Y R_{z} Y+ \\
& (I-Y X) Q_{y}(I-X Y) .
\end{aligned}
$$

A simple inspection shows that $Z=(I-Y X)^{-1} Y \geq 0$ (since $Y \geq 0$ and $\rho(Y X)<1$ ) satisfies the ARE

$$
A_{z} Z+Z A_{z}^{T}+Z R_{z} Z+Q_{y}=0 .
$$

Furthermore, $Z$ is the stabilizing solution of the ARE (18) since it can be shown that $A_{z}+Z R_{z}=$ $(I-Y X)^{-1}\left(A_{y}+Y R_{y}\right)(I-Y X)$, i.e.

$$
\sigma\left(A_{z}+Z R_{z}\right) \subset \mathbb{C}_{<0} .
$$

Also, long but routine algebraic manipulations show that

$$
\begin{aligned}
A_{z}= & \bar{A}-B_{1} R^{-1} \bar{C}_{1}-B_{1} R^{-1} D_{21}^{T}\left(D_{21} R^{-1} D_{21}^{T}\right)^{-1} \\
& \left(\bar{C}_{2}-D_{21} R^{-1} \bar{C}_{1}\right), \\
R_{z}= & \bar{C}_{1}^{T} R^{-1} \bar{C}_{1}-\left(\bar{C}_{2}-D_{21} R^{-1} \bar{C}_{1}\right)^{T}\left(D_{21} R^{-1} D_{21}^{T}\right)^{-1} \\
& \left(\bar{C}_{2}-D_{21} R^{-1} \bar{C}_{1}\right),
\end{aligned}
$$

where $\bar{A}=A-B_{1} R^{-1} C_{E}, \bar{C}_{1}=-C_{1} B_{2} F, \bar{C}_{2}=C_{2}-$ $D_{21} R^{-1} C_{E}$ and $C_{E}=C_{1} A_{F}-B_{1}^{T} X$. Thus, by replacing 
(20), (21) and $Q_{y}$ in the $\operatorname{ARE~(18),~we~get~}$

$$
\begin{aligned}
0= & A_{z} Z+Z A_{z}^{T}+Z R_{z} Z+Q_{y} \\
= & \bar{A} Z+Z \bar{A}^{T}+\left(B_{1}-Z \bar{C}_{1}^{T}\right) R^{-1}\left(B_{1}-Z \bar{C}_{1}^{T}\right)^{T}-\left[B_{1}\right. \\
& \left.R^{-1} D_{21}^{T}+Z\left(\bar{C}_{2}-D_{21} R^{-1} \bar{C}_{1}\right)^{T}\right]\left(D_{21} R^{-1} D_{21}^{T}\right)^{-1} \\
& {\left[B_{1} R^{-1} D_{21}^{T}+Z\left(\bar{C}_{2}-D_{21} R^{-1} \bar{C}_{1}\right)^{T}\right]^{T} } \\
= & \left(\bar{A}+\bar{L} \bar{C}_{2}\right) Z+Z\left(\bar{A}+\bar{L} \bar{C}_{2}\right)^{T}+\left(B_{1}+\bar{L} D_{21}-\right. \\
& \left.Z \bar{C}_{1}^{T}\right) R^{-1}\left(B_{1}+\bar{L} D_{21}-Z \bar{C}_{1}^{T}\right)^{T},
\end{aligned}
$$

where $\bar{L}=-\left[B_{1} R^{-1} D_{21}^{T}+Z\left(\bar{C}_{2}-D_{21} R^{-1} \bar{C}_{1}\right)^{T}\right]$ $\left(D_{21} R^{-1} D_{21}^{T}\right)^{-1}$. In addition, it can be shown that $\bar{A}+\bar{L} \bar{C}_{2}-\left(B_{1}+\bar{L} D_{21}-Z \bar{C}_{1}^{T}\right) R^{-1} \bar{C}_{1}=A_{z}+Z R_{z}$, so it follows from (19) that

$$
\sigma\left(\bar{A}+\bar{L} \bar{C}_{2}-\left(B_{1}+\bar{L} D_{21}-Z \bar{C}_{1}^{T}\right) R^{-1} \bar{C}_{1}\right) \subset \mathbb{C}_{<0}
$$

Therefore, since $R=R^{T}>0$ (by assumption) and $Z=$ $Z^{T} \geq 0$ is the stabilizing solution of (22), it follows, by $[27$, Lemma 2.3], that the (output-injection) system

$$
\left[\begin{array}{c|c}
\bar{A}+\bar{L}_{2} \bar{C}_{2} & B_{1}+\bar{L} D_{21} \\
\hline \bar{C}_{1} & C_{1} B_{1}
\end{array}\right]
$$

is SPR with $\bar{A}+\bar{L} \bar{C}_{2}$ Hurwitz and the ARE

$$
A_{L}^{T} W+W A_{L}+\left(\bar{C}_{1}-B_{1 L}^{T} W\right)^{T} R^{-1}\left(\bar{C}_{1}-B_{1 L}^{T} W\right)=0
$$

has a real stabilizing solution $W=W^{T} \geq 0$, i.e.

$$
\sigma\left(A_{L}-B_{1 L} R^{-1}\left(\bar{C}_{1}-B_{1 L}^{T} W\right)\right) \subset \mathbb{C}_{<0},
$$

where $A_{L}=\bar{A}+\bar{L} \bar{C}_{2}$ and $B_{1 L}=B_{1}+\bar{L} D_{21}$.

Finally, let $\mathcal{Q}(\mathcal{X})=\left[\begin{array}{ll}Q_{11} & Q_{21}^{T} \\ Q_{21} & Q_{22}\end{array}\right]$ with $\mathcal{X}=\left[\begin{array}{cc}X & 0 \\ 0 & W\end{array}\right]$. It is shown after some calculations that

$$
\begin{aligned}
Q_{11}= & A_{F}^{T} X+X A_{F}+\left(C_{1} A_{F}-B_{1}^{T} X\right)^{T} R^{-1} \\
& \left(C_{1} A_{F}-B_{1}^{T} X\right)=0 \\
Q_{21}= & -F^{T} B_{2}^{T}\left(X+C_{1}^{T} R^{-1} C_{E}\right)=0 \\
Q_{22}= & A_{L}^{T} W+W A_{L}+\left(C_{1} B_{2} F+B_{1 L}^{T} W\right)^{T} R^{-1} \\
& \left(C_{1} B_{2} F+B_{1 L}^{T} W\right)=0 .
\end{aligned}
$$

In other words, $\mathcal{X}=\mathcal{X}^{T} \geq 0$ satisfies (16), so via [24, Lemma 2], we conclude that $\mathcal{F}_{l}(G, K)$ is NI.

Moreover, let $A_{c}-B_{c} R^{-1}\left(C_{c} A_{c}-B_{c}^{T} \mathcal{X}\right)=\left[\begin{array}{ll}\mathcal{A}_{11} & \mathcal{A}_{12} \\ \mathcal{A}_{21} & \mathcal{A}_{22}\end{array}\right]$.
Then,

$$
\begin{aligned}
& \mathcal{A}_{11}=A_{F}-B_{1} R^{-1}\left(C_{1} A_{F}-B_{1}^{T} X\right) \\
& \mathcal{A}_{21}=-\Psi+\Psi=0 \\
& \mathcal{A}_{22}=A_{L}+B_{1 L} R^{-1}\left(C_{1} B_{2} F+B_{1 L}^{T} W\right)
\end{aligned}
$$

and consequently,

$$
\sigma\left(A_{c}-B_{c} R^{-1}\left(C_{c} A_{c}-B_{c}^{T} \mathcal{X}\right)\right)=\sigma\left(\mathcal{A}_{11}\right) \cup \sigma\left(\mathcal{A}_{22}\right) .
$$

Since $\sigma\left(\mathcal{A}_{22}\right) \subset \mathbb{C}_{<0}$ and $\mathcal{A}_{11}=A_{x}+R_{x} X$ (as shown in Theorem 5.1), $\sigma\left(A_{c}-B_{c} R^{-1}\left(C_{c} A_{c}-B_{c}^{T} \mathcal{X}\right)\right) \subset \mathbb{C}_{<0} \cup\{0\}$ when $\sigma\left(A_{x}+R_{x} X\right) \subset \mathbb{C}_{<0} \cup\{0\}$. Then, via Theorem 3.1, we conclude that (whenever $\left.\operatorname{det}\left(A_{c}\right) \neq 0\right), \mathcal{F}_{l}(G, K)$ is SNI and the associated linear fractional interconnection is internally stable.

Remark 5.5. The procedure to synthesize a controller for the SNI problem is as follows: 1) verify that $D_{11}$ is symmetric, 2) find the unique solution of the ARE in condition (iii) in Theorem 5.4, 3) find all the solutions to the ARE in condition (ii) of Theorem $5.4,4)$ from all the solutions found in step 3) check which of them satisfy $\sigma\left(A_{x}+R_{x} X\right) \subset \mathbb{C}_{<0} \cup\{0\}$, $\operatorname{det}\left(\left[\begin{array}{cc}A+B_{2} F & -B_{2} F \\ -\Psi & A+\Psi-B_{k} C_{2}\end{array}\right]\right) \neq 0$ and condition (iv) of Theorem 5.4. It is worth mentioning that there may be more than one controller which solves this problem, as pointed out in Remark 5.2.

\section{Illustrative example}

Consider the uncertain system in Fig. 1, where the plant $G(s)$ is described by

$$
\left[\begin{array}{l}
z(t) \\
y(t)
\end{array}\right]=\left[\begin{array}{ccc|cc}
-1 & 0 & 0 & -1 & 0 ! 0 \\
1 & -1 & 1 & 0 & 1: 0 \\
0 & 1 & -1 & 0 & 1: 1 \\
\hline-1 & 0 & 0 & 1 & 0 ! 0 \\
0 & 1 & 1 & 0 & 1: 0 \\
\hdashline & -1 & 0 & -1 & 0 \\
1 & -1 & 0 \\
0 & 1 & 1 & 1 & 2 ! 0
\end{array}\right]\left[\begin{array}{l}
w(t) \\
u(t)
\end{array}\right]
$$

It can be easily seen that the assumptions of Theorem 5.4 are fulfilled. It is also easy to check that for any $\alpha \geq 0$, the matrices $X=\left[\begin{array}{lll}1 & 0 & 0 \\ 0 & \alpha & \alpha \\ 0 & \alpha & \alpha\end{array}\right] \geq 0$ and $Y=0$ satisfy the conditions (i) to (iv) of Theorem 5.4 and $\sigma\left(A_{x}+R_{x} X\right)=$ $\{0,0,-2\}$. We also construct $F=-\left[\begin{array}{lll}1 & 2 \alpha & 2 \alpha\end{array}\right], L=$ 
$-\left[\begin{array}{cc}1 & 0 \\ 0.5 & 0.5 \\ 0.5 & 0.5\end{array}\right], \Psi=0, A_{c}=\left[\begin{array}{c:ccc}A+B_{2} F & -B_{2} F \\ 0 & A+L C_{2}\end{array}\right]=$
$\left[\begin{array}{ccc:ccc}-1 & 0 & 0 & 0 & 0 & 0 \\ 1 & -1 & 1 & 0 & 0 & 0 \\ -1 & 1-2 \alpha & -2 \alpha-1 & 1 & 2 \alpha & 2 \alpha \\ \hdashline 0 & 0 & 0 & -2 & 1 & 0 \\ 0 & 0 & 0 & 0.5 & -1 & 0.5 \\ 0 & 0 & 0 & -0.5 & 1 & -1.5\end{array}\right]$ and a controller
$K(s)$ is given by

$$
u(t)=\left[\begin{array}{ccc|cc}
-2 & 1 & 0 & 1 & 0 \\
0.5 & -1 & 0.5 & 0.5 & 0.5 \\
-1.5 & 1-2 \alpha & -1.5-2 \alpha & 0.5 & 0.5 \\
\hline-1 & -2 & -2 & 0 & 0
\end{array}\right] y(t)
$$

It is easy to verify that the modes of the closed-loop system matrix $A_{c}$ are $\{-2.618,-2,-1.5,-1,-0.382,-2 \alpha\}$ and $\operatorname{det}\left(A_{c}\right)=6 \alpha$. Furthermore, the modes $\sigma(A+$ $\left.L C_{2}\right)=\{-2.618,-1.5,-0.382\}$ are uncontrollable since $B_{c}=\left[\begin{array}{ll}B_{1}^{T} & 0\end{array}\right]^{T}$ and the mode $\lambda=-2$ is unobservable. Hence, the closed-loop poles are given by $\{-1,-2 \alpha\}$, the closed-loop transfer function matrix is given by $\mathcal{F}_{l}(G, K)(s)=\left[\begin{array}{cc}\frac{1}{s+1}+1 & 0 \\ 0 & \frac{2}{s+2 \alpha}+1\end{array}\right]$ and $j\left[\mathcal{F}_{l}(G, K)(j \omega)-\mathcal{F}_{l}(G, K)(j \omega)^{*}\right]=\left[\begin{array}{cc}\frac{2 \omega}{\omega^{2}+1} & 0 \\ 0 & \frac{4 \omega}{4 \alpha^{2}+\omega^{2}}\end{array}\right]$.

Note that for all $\alpha>0$, $\operatorname{det}\left(A_{c}\right) \neq 0$ and therefore Theorem 5.4 states that $\mathcal{F}_{l}(G, K)$ is SNI and its associated linear fractional interconnection is internally stable. To verify this, note that none of the poles of $\mathcal{F}_{l}(G, K)$ and also none of the modes of $A_{c}$ are in $\{s \in \mathbb{C}: \Re(s) \geq 0\}$ and $j\left[\mathcal{F}_{l}(G, K)(j \omega)-\mathcal{F}_{l}(G, K)(j \omega)^{*}\right]>0$ for all $\omega>0$.

Moreover, for $\alpha=0, \operatorname{det}\left(A_{c}\right)=0$. Thus, $\mathcal{F}_{l}(G, K)=$ $\left[\begin{array}{cc}\frac{1}{s+1}+1 & 0 \\ 0 & \frac{2}{s}+1\end{array}\right]$ is clearly only NI with a pole at the origin. To verify this, note that

(i) the poles of $\mathcal{F}_{l}(G, K)$ are $\{-1,0\}$ none of which is in $\{s \in \mathbb{C}: \Re(s)>0\}$;

(ii) $j\left[\mathcal{F}_{l}(G, K)(j \omega)-\mathcal{F}_{l}(G, K)(j \omega)^{*}\right] \geq 0 \quad \forall \omega>0$;

(iii) there are no poles on $s=j \omega_{0}$ for $\omega_{0} \in(0, \infty)$;

(iv) $s=0$ is a pole of $\mathcal{F}_{l}(G, K)$ but $\lim _{s \rightarrow 0} s^{2} \mathcal{F}_{l}(G, K)(s)$ $=0 \geq 0$.

\section{Conclusions}

A set of necessary and sufficient conditions were established for the characterization of SNI systems with nonnecessarily minimal state-space realization, which we refer to as the SNI Lemma. This SNI lemma then allowed us to build on prior methods for SPR synthesis to develop an ARE based method for SNI controller synthesis. The proposed methodology involves the solution of two AREs and a coupling condition, but due to essential peculiarities only present in SNI controller synthesis, one of these AREs has a singular Hamiltonian. This critical solution may not be unique, as shown in the illustrative example; therefore, its construction can be numerically challenging. Although the sufficient conditions provided for SNI controller synthesis do not appear to be conservative, necessary conditions for SNI controller synthesis remain an open problem.

\section{References}

[1] H. Abou-Kandil, G. Freiling, V. Ionescu, and G. Jank. Matrix Riccati Equations in Control and Systems Theory. Birkhäuser Basel, 1 edition, 2003.

[2] D. A. Bini, B. Iannazzo, and B. Meini. Numerical Solution of Algebraic Riccati Equations. Society for Industrial and Applied Mathematics, Philadelphia, PA, USA, 2012.

[3] H. J. Chen and A. Lanzon. Closed-loop stability analysis of discrete-time negative imaginary systems. Systems 8 Control Letters, 114:52-58, Apr 2018.

[4] J. C. Doyle, K. Glover, P. P. Khargonekar, and B. A. Francis. State-space solutions to standard $\mathcal{H}_{2}$ and $\mathcal{H}_{\infty}$ control problems. IEEE Transactions on Automatic Control, 34(8):831-847, 1989.

[5] A. Ferrante, A. Lanzon, and L. Ntogramatzidis. Foundations of not necessarily rational negative imaginary systems theory: Relations between classes of negative imaginary and positive real systems. IEEE Transactions on Automatic Control, 61(10):3052-3057, Oct 2016.

[6] A. Ferrante, A. Lanzon, and L. Ntogramatzidis. Discretetime negative imaginary systems. Automatica, 79:1-10, May 2017.

[7] V. Ionescu, C. Oara, and M. D. Weiss. Generalized Riccati Theory and Robust Control: A Popov Function Approach. Wiley, 1999.

[8] P. Lancaster and L. Rodman. Algebraic Riccati Equations. Clarendon Press, Oxford, UK, 1995.

[9] A. Lanzon and H. J. Chen. Feedback stability of negative imaginary systems. IEEE Transactions on Automatic Control, 62(11):5620-5633, Nov 2017.

[10] A. Lanzon and I. R. Petersen. Stability robustness of a feedback interconnection of systems with negative imaginary frequency response. IEEE Transactions on Automatic Control, 53(4):1042-1046, May 2008.

[11] A. Lanzon, Z. Song, S. Patra, and I. R. Petersen. A strongly strict negative-imaginary lemma for non-minimal linear systems. Communications in Information and Systems, 11(2):139-152, 2011.

[12] V. Larin. Algorithm for solving algebraic Riccati equation which has singular Hamiltonian matrix. Systems \& Control Letters, 36:231-239, 1999. 
[13] K. Z. Liu, M. Ono, X. Huo, and M. Wu. Robust performance design for systems with output strictly passive uncertainty. IEEE Transactions on Industrial Electronics, 65(5):42074215, May 2018.

[14] K. Z. Liu, M. Ono, X. Li, and M. Wu. Robust performance synthesis for systems with positive-real uncertainty and an extension to the negative-imaginary case. Automatica, 82:194-201, Aug 2017.

[15] M. Liu and J. Xiong. On non-proper negative imaginary systems. Systems \& Control Letters, 88:47-53, Feb 2016.

[16] M. Liu and J. Xiong. Properties and stability analysis of discrete-time negative imaginary systems. Automatica, 83:58-64, Sep 2017.

[17] M. Liu and J. Xiong. Bilinear transformation for discretetime positive real and negative imaginary systems. IEEE Transactions on Automatic Control, 63(12):4264-4269, Dec 2018.

[18] M. Mabrok, A. G. Kallapur, I. R. Petersen, and A. Lanzon. Generalizing negative imaginary systems theory to include free body dynamics: Control of highly resonant structures with free body motion. IEEE Transactions on Automatic Control, 59(10):2692-2707, Oct 2014.

[19] M. Mabrok, A. G. Kallapur, I. R. Petersen, and A. Lanzon. Spectral conditions for negative imaginary systems with applications to nanopositioning. IEEE/ASME Transactions on Mechatronics, 19(3):895-903, Jun 2014.

[20] M. Mabrok, A. G. Kallapur, I. R. Petersen, and A. Lanzon. A generalized negative imaginary lemma and Riccati-based static state-feedback negative imaginary synthesis. Systems \&) Control Letters, 77:63-68, Mar 2015.

[21] S. Patra and A. Lanzon. Stability analysis of interconnected systems with "mixed" negative-imaginary and small-gain properties. IEEE Transactions on Automatic Control, 56(6):1395-1400, Jun 2011.

[22] I. R. Petersen. Negative imaginary systems theory and applications. Annual Reviews in Control, 42:309-318, 2016.

[23] I. R. Petersen and A. Lanzon. Feedback control of negativeimaginary systems. IEEE Control Systems Magazine, 30(5):54-72, Oct 2010.

[24] G. Salcan-Reyes and A. Lanzon. On negative imaginary synthesis via solutions to Riccati equations. In Proceedings of the 16th European Control Conference, pages 870-875, Limassol, Cyprus, June 2018.

[25] Z. Song, A. Lanzon, S. Patra, and I. R. Petersen. Towards controller synthesis for systems with negative imaginary frequency response. IEEE Transactions on Automatic Control, 55(6):1506-1511, Jun 2010.

[26] Z. Song, A. Lanzon, S. Patra, and I. R. Petersen. A negative-imaginary lemma without minimality assumptions and robust state-feedback synthesis for uncertain negativeimaginary systems. Systems $\&$ Control Letters, 61:1269-1276, Dec 2012.

[27] W. Sun, P. P. Khargonekar, and D. Shim. Solution to the positive real control problem for linear timeinvariant systems. IEEE Transactions on Automatic Control, 39(10):2034-2046, Oct 1994.

[28] J. Xiong, J. Lam, and I. R Petersen. Output feedback negative imaginary synthesis under structural constraints. Automatica, 71:222-228, Sep 2016.

[29] J. Xiong, A. Lanzon, and I. R Petersen. Descriptor systems state-space conditions to guarantee negative imaginary properties without minimality restrictions. In Proceedings of the 54th IEEE Conference on Decision and Control, pages 2707-2712, Osaka, Japan, 2015.
[30] J. Xiong, I. R. Petersen, and A. Lanzon. A negative imaginary lemma and the stability of interconnections of linear negative imaginary systems. IEEE Transactions on Automatic Control, 55(10):2342-2347, Oct 2010.

[31] K. Zhou, J. C. Doyle, and K. Glover. Robust and Optimal Control. Prentice Hall, Upper Saddle River, NJ, USA, 1996. 